\title{
NORM CLOSURE OF CLASSICAL PSEUDODIFFERENTIAL OPERATORS DOES NOT CONTAIN HÖRMANDER'S CLASS
}

\author{
SEVERINO T. MELO
}

\begin{abstract}
The $L^{2}$-norm closure $\mathfrak{A}_{c l}$ of the set $\Psi_{c l}^{0}(X)$ of all zero-order classical pseudodifferential operators on a closed manifold $X$ is proven not to contain Hörmander's class $\Psi^{0}(X)$. A set of generators for $\mathfrak{A}_{c l}$ smaller than all of $\Psi_{c l}^{0}(X)$ is also described.
\end{abstract}

\section{INTRODUCTION}

Let $X$ denote a closed manifold of dimension $n$. As in his book [9], we denote by $\Psi^{0}(X)$ the set of all zero-order elements in Hörmander's class of pseudodifferential operators (of type $\rho=1$ and $\delta=0$ ). The subclass of classical pseudodifferential operators of order zero, i.e., those whose local symbols possess asymptotic expansions in homogeneous components of decreasing degree, will be here denoted by $\Psi_{c l}^{0}(X)$ (this is denoted by $\Psi_{p h g}^{0}(X)$ in $[9]$ ).

The Hilbert space of square integrable functions over $X$ (equipped with a measure defined by a positive smooth density) will be denoted by $\mathfrak{H}$, and its algebra of bounded operators by $\mathfrak{L}(\mathfrak{H})$. The classes of pseudodifferential operators $\Psi^{0}(X)$ and $\Psi_{c l}^{0}(X)$ are adjoint-invariant subalgebras of $\mathfrak{L}(\mathfrak{H})$. Their norm closures will be denoted by $\mathfrak{A}$ and $\mathfrak{A}_{c l}$, respectively. Both contain the ideal $\mathfrak{K}$ of compact operators, and their quotients by it are unital commutative $\mathrm{C}^{*}$-algebras; hence isomorphic to spaces of continuous functions over compact Hausdorff spaces. That $\mathfrak{A}$ is not contained in $\mathfrak{A}_{c l}$ follows from the fact that these spaces, the Gelfand spaces of $\mathfrak{A} / \mathfrak{K}$ and of $\mathfrak{A}_{c l} / \mathfrak{K}$, are not the same; as we prove in Sections 2 and 3

Our proofs strongly depend on Hörmander's estimate for the norm, modulo compact operators, of a pseudodifferential operator (8], Theorem 3.3). For classical pseudodifferential operators, that estimate had already appeared in [10, Theorem A.4; and had been proven, for singular integral operators, by Gohberg [7] and Seeley [15].

In Section 4 we show that $\mathfrak{A}_{c l}$ is actually a comparison algebra, as defined by Cordes 4]. This means that it is equal to the smallest $\mathrm{C}^{*}$-subalgebra of $\mathfrak{L}(\mathfrak{H})$ containing all operators of multiplication by smooth functions and all operators of the form $L(1-\Delta)^{-1 / 2}$, where $L$ is any first-order linear differential operator with smooth coefficients and $\Delta$ is a certain second-order non-positive elliptic differential operator on $X$. That can be proven again by a Gelfand space argument.

On page 508 in the first of a series of papers about their index theorem, Atiyah and Singer [1] state: "The term pseudodifferential operator is applied, in different places, to slightly different classes of operators. For our purposes any one of these classes would be equally good. In fact, we shall eventually form a closure of this class and, by that stage, any differences would disappear. Perhaps the largest and most natural class is that given by Hörmander [8] (...)". The norm closure of the 
set of all pseudodifferential operators considered by Atiyah and Singer in [1] (the subclass of Hörmander's class defined on page 509) is however smaller than the closure of the class introduced in [8]; whose zero-order subclass was later denoted $\Psi^{0}(X)$. Indeed, the results in Section 3 show, for example, that the image of the continuous extension of the principal symbol to the closure of $\Psi^{0}(X)$ cannot be identified with the space of continuous functions on the co-sphere bundle, as described in the Remark right after (5.2) in 1 .

Taylor proved [16] that a bounded operator $A$ on $L^{2}\left(\mathbb{S}^{n}\right)$ belongs to $\Psi^{0}\left(\mathbb{S}^{n}\right)$ if and only if the mapping

$$
S O_{e}(n+1,1) \ni g \mapsto U(g) A U(g)^{-1} \in \mathfrak{L}\left(L^{2}\left(\mathbb{S}^{n}\right)\right)
$$

is smooth, where $U$ denotes the unitary representation of the conformal group $S O_{e}(n+1,1)$ on $L^{2}\left(\mathbb{S}^{n}\right)$ induced by its natural action on the sphere $\mathbb{S}^{n}$ (for simplicity, only this particular case of his result is stated here). The fact that the norm closure of the classical pseudodifferential operators is smaller than that of Hörmander's class makes it plausible to expect that, perhaps, the classical pseudodifferential operators can also be characterized by a similar smoothness condition, for a larger group. On the other direction, the class of smooth operators for the action of (the subgroup of the conformal group) $S O(n)$ contains operators without the pseudolocal property [6, 11]. We refer to [13] for possible applications of these smoothness characterizations of pseudodifferential operators, the first of which was given by Cordes [2].

\section{The Gelfand Space of $\mathfrak{A} / \mathfrak{K}$}

The "basic calculus" (see [9], Section 18.1; or [17, Sections I.3 and I.4) of pseudodifferential operators implies that the commutator of any two elements of $\Psi^{0}(X)$ is of order -1 . Operators of order -1 are bounded from $\mathfrak{H}$ into the Sobolev space $H^{1}(X)$, which compactly embeds in $\mathfrak{H}$. Since $\Psi^{0}(X)$ is dense in $\mathfrak{A}$, all commutators of $\mathfrak{A}$ then belong to the compact ideal $\mathfrak{K}$.

Any integral operator with smooth kernel is a pseudodifferential operator of order $-\infty$ on $X$. In particular, any finite rank operator with image and kernel contained in $C^{\infty}(X)$ belongs to $\Psi^{0}(X)$. A simple density argument gives that all finite rank operators belong to $\mathfrak{A}$, and then $\mathfrak{K} \subset \mathfrak{A}$. The quotient $\mathfrak{A} / \mathfrak{K}$ is therefore commutative. It is, moreover, a $\mathrm{C}^{*}$-algebra (12, 3.1.4).

Any unital commutative $\mathrm{C}^{*}$-algebra $\mathfrak{B}$ is isomorphic to the algebra of continuous functions on the set $\mathbb{M}$ of its nonzero multiplicative linear functionals (which are automatically continuous of norm 1) equipped with the weak-* topology. This is Gelfand's theorem (12, 2.1.10), and we will call $\mathbb{M}$ the Gelfand space of $\mathfrak{B}$. That theorem also states that the Gelfand mapping for $\mathfrak{B}$,

$$
\begin{aligned}
\mathfrak{B} & \longrightarrow C(\mathbb{M}) \\
x & \longmapsto(\mathbb{M} \ni \varphi \mapsto \varphi(x)),
\end{aligned}
$$

is an isomorphism.

In this Section, we show that the Gelfand space of $\mathfrak{A} / \mathfrak{K}$ are the points at infinity of the compactification of the cotangent bundle determined by the zero-order symbols, and that the Gelfand mapping then corresponds to the restriction of the principal symbol over those points.

Let $\mathfrak{S}$ denote the closure in sup-norm of $S^{0}\left(T^{*} X\right)$, the set of symbols of order zero on the cotangent bundle $T^{*} X$ (as defined in [9], page 85 ). This is a $\mathrm{C}^{*}$-subalgebra 
of the set of all continuous bounded functions on $T^{*} X$. Let $\mathbb{M}_{s}$ denote the Gelfand space of $\mathfrak{S}$. Evaluation at each point defines a mapping from $T^{*} X$ to $\mathbb{M}_{s}$. It follows from the definition of weak-* topology that this mapping is continuous. It follows from Urysohn's Lemma that it is a homeomorphism onto its dense image $\left(\mathbb{M}_{s}\right.$ is a compact Hausdorff space). In other words, $\mathbb{M}_{s}$ is a compactification of $T^{*} X$. Regarding this embedding of $T^{*} X$ into $\mathbb{M}_{s}$ as an inclusion, let us define

$$
\mathbb{M}_{h}=\mathbb{M}_{s} \backslash T^{*} X
$$

The unique continuous extension of any $a \in S^{0}\left(T^{*} X\right)$ to $\mathbb{M}_{s}$ will also be denoted by $a$.

Lemma 1. Any symbol of order -1 , a $\in S^{-1}\left(T^{*} X\right)$, vanishes on $\mathbb{M}_{h}$.

Proof: Given $m \in \mathbb{M}_{h}$, there is a net $v_{\alpha} \in T^{*} X$ converging to $m$. Denoting by $\pi: T^{*} X \rightarrow X$ the bundle projection and, if necessary, passing to a subnet, we may suppose that every $x_{\alpha}=\pi\left(v_{\alpha}\right) \in X$ belongs to the domain of a chart $\chi: U \rightarrow \tilde{U}$ and that $x_{\alpha}$ converges to an $x_{0} \in U$ (we have used that any net in the compact space $X$ has a convergent subnet; see [14, Theorem IV.3).

Let $\phi: T^{*} U \rightarrow \tilde{U} \times \mathbb{R}^{n}$ denote the bundle trivialization induced by $\chi$, and let $\phi\left(v_{\alpha}\right)=\left(y_{\alpha}, \xi_{\alpha}\right)$. I claim that $\left|\xi_{\alpha}\right| \rightarrow \infty$. Indeed, if that were not true, $\xi_{\alpha}$ would have a subnet $\xi_{F(\alpha)}$ converging to some $\xi_{0} \in \mathbb{R}^{n}$. Then $v_{F(\alpha)}$ would converge to $\phi^{-1}\left(\chi\left(x_{0}\right), \xi_{0}\right)$. That would contradict the assumption that $\lim v_{\alpha}$ is a point in $\mathbb{M}_{h}=\mathbb{M}_{s} \backslash T^{*} X$.

The pushforward of $a$ by $\phi$ is, by definition, a local symbol of order $-1, \phi_{*} a \in$ $S^{-1}\left(\tilde{U} \times \mathbb{R}^{n}\right)$. It also no loss of generality to suppose that the net with which we started is such that there is a compact subset of $\tilde{U}$ containing every $y_{\alpha}$; and then $\phi_{*} a\left(y_{\alpha}, \xi_{\alpha}\right)=a\left(\phi^{-1}\left(y_{\alpha}, \xi_{\alpha}\right)\right)$ converges to zero. This proves that $a(m)=$ $\lim a\left(v_{\alpha}\right)=0$.

Lemma 2. For all $a \in S^{0}\left(T^{*} X\right)$ we have:

$$
\sup \left\{|a(m)| ; m \in \mathbb{M}_{h}\right\}=\lim _{R \rightarrow \infty} \sup \left\{|a(v)| ; v \in T^{*} X,|v|^{\prime}>R\right\},
$$

where $|v|^{\prime}$ denotes the evaluation at $v$ of the norm defined on $T_{\pi(v)}^{*} X$ by a Riemannian metric on $X$.

Proof: Since $\mathbb{M}_{h}$ is compact, there is an $m_{0} \in \mathbb{M}_{h}$ such that $a\left(m_{0}\right)$ equals the left-hand side of (1), which we denote by $\left\|\left.a\right|_{\mathbb{M}_{h}}\right\|_{\infty}$. Let $v_{\alpha}$ denote a net in $T^{*} X$ which converges to $m_{0}$. We may suppose, as in the proof of Lemma 11 that $v_{\alpha}$ is locally given by $\left(y_{\alpha}, \xi_{\alpha}\right)$, with $\left|\xi_{\alpha}\right| \rightarrow \infty,|\cdot|$ denoting the euclidean norm on $\mathbb{R}^{n}$. It is easy to see that $\left|v_{\alpha}\right|^{\prime} \rightarrow \infty$. If we define $R_{\alpha}=\left|v_{\alpha}\right|^{\prime} / 2$, we then have $\lim _{\alpha} R_{\alpha}=\infty$. Since

$$
\left|a\left(v_{\alpha}\right)\right| \leq \sup \left\{|a(v)| ;|v|^{\prime}>R_{\alpha}\right\}
$$

we have:

$$
\begin{gathered}
\left.|| a\right|_{\mathbb{M}_{h}} \|_{\infty}=\lim _{\alpha}\left|a\left(v_{\alpha}\right)\right| \leq \\
\lim _{\alpha} \sup \left\{|a(v)| ;|v|^{\prime}>R_{\alpha}\right\}=\lim _{R \rightarrow \infty} \sup \left\{|a(v)| ;|v|^{\prime}>R\right\} .
\end{gathered}
$$

Suppose the above inequality is strict. Then there is $\delta>0$ and a sequence $v_{k} \in T^{*} X$ such that $\left|a\left(v_{k}\right)\right|$ converges to $\left.|| a\right|_{\mathbb{M}_{h}} \|+\delta$ and $\left|v_{k}\right|^{\prime} \rightarrow \infty$. Let $v_{\alpha}$ be a convergent subnet of that sequence. Its limit $m_{0}$ cannot belong to $T^{*} X$, 
since $\left|v_{\alpha}\right|^{\prime} \rightarrow \infty$. That gives $\left|a\left(m_{0}\right)\right|>\left\|\left.a\right|_{\mathbb{M}_{h}}\right\|_{\infty}$ for some $m_{0} \in \mathbb{M}_{h}$, which is a contradiction.

The principal symbol of an operator in $\Psi^{0}(X)$ is defined modulo negative-order terms (9, pages 86 and 87). That gives a surjective homomorphism from $\Psi^{0}(X)$ onto $S^{0}\left(T^{*} X\right) / S^{-1}\left(T^{*} X\right)$. By Lemma 1 the restrictions to $\mathbb{M}_{h}$ of any two representatives of the principal symbol of a given $A \in \Psi^{0}(X)$ coincide. This defines a mapping

$$
\sigma: \Psi^{0}(X) \longrightarrow C\left(\mathbb{M}_{h}\right)
$$

which is an algebra homomorphism (9], Theorem 18.1.23). It also follows from the basic calculus that $\sigma\left(A^{*}\right)=\overline{\sigma(A)}$, for all $A \in \Psi^{0}(X)$, with $A^{*}$ denoting the Hilbert-space adjoint of $A$, and bar denoting complex conjugate.

Theorem 1. The ${ }^{*}$-homomorphism $\sigma$ is bounded. Its continuous extension to $\mathfrak{A}$, still denoted by $\sigma$, is surjective and has kernel equal to the compact ideal $\mathfrak{K}$.

Proof: The proof of [8], Theorem 3.3 (see the Remark at the end of that proof), shows that, for every $A \in \Psi^{0}(X)$,

$$
\inf \{\|A+K\| ; K \in \mathfrak{K}\}=\lim _{R \rightarrow \infty} \sup \left\{|a(v)| ; v \in T^{*} X,|v|^{\prime}>R\right\},
$$

which equals $\left\|\left.a\right|_{\mathbb{M}_{h}}\right\|_{\infty}$, by Lemma 2

Since the left-hand side of (2) is bounded by $\|A\|$, we obtain that $\sigma$ extends to a bounded operator between the Banach spaces $\mathfrak{A}$ and $C\left(\mathbb{M}_{h}\right)$. It also follows from (2) that the kernel of that extension is equal to $\mathfrak{K}$, and that the mapping induced on the quotient is an isometry; and hence has closed image. By definition, $\left\{\sigma(A) ; A \in \Psi^{0}(X)\right\}$ is dense in $C\left(\mathbb{M}_{h}\right)$, and hence $\sigma$ is onto.

Corollary 1. The mapping

$$
\begin{aligned}
\mathfrak{A} / \mathfrak{K} & \longrightarrow C\left(\mathbb{M}_{h}\right) \\
{[A] } & \longmapsto \sigma(A)
\end{aligned}
$$

is a $C^{*}$-algebra isomorphism $([A]$ denotes the class of $A \in \mathfrak{A}$ in the quotient $\mathfrak{A} / \mathfrak{K})$.

\section{The Gelfand SPACE of $\mathfrak{A}_{c l} / \mathfrak{K}$}

An operator $A \in \Psi^{0}(X)$ is classical, or polihomogeneous, if for every chart $\chi$ : $U \rightarrow \tilde{U}$, the pushforward $\chi_{*} A$,

$$
C_{0}^{\infty}(\tilde{U}) \ni u \mapsto\left(\chi_{*} A\right) u=[A(u \circ \chi)] \circ \chi^{-1} \in C^{\infty}(\tilde{U}),
$$

is equal, modulo an integral operator with smooth kernel, to $\tilde{a}(x, D)$, for some $\tilde{a} \in S^{0}\left(\tilde{U} \times \mathbb{R}^{n}\right)$ possessing an asymptotic expansion

$$
\tilde{a}(x, \xi) \sim \sum_{j=0}^{\infty} a_{j}(x, \xi)
$$

where each $a_{j}$ satisfies $a_{j}(x, t \xi)=t^{-j} a_{j}(x, \xi)$, for every $t>0$ and every $\xi$ with $|\xi|>1$. Although $\tilde{a}$ and $a_{j}$ for $j>0$ are only locally defined, $a_{0}$ is a globally defined function on the cotangent bundle (see, for example, the comments at the end of Section I.5 in [17). That gives a representative of the principal symbol of $A$ satisfying, for some $R_{0}>0$,

$$
a(t v)=a(v), \text { if } t>0 \text { and }|v|^{\prime}>R_{0} .
$$


Moreover, it is possible to arrange $R_{0}$ to be smaller than one. For such an $a$, $\sup \left\{|a(v)| ;|v|^{\prime}>R\right\}$ is independent of $R$, for $R \geq 1$. If $a_{1}$ is another principal symbol of $A$ satisfying (3) with $R_{0}<1$, then $a_{1}$ and $a$ coincide on $\left\{|v|^{\prime} \geq 1\right\}$, by Lemmas 1 and 2. The following theorem then follows from the estimate (2).

Theorem 2. If $A \in \Psi^{0}(X)$ is classical (denoted $A \in \Psi_{c l}^{0}(X)$ ) and $a \in C^{\infty}\left(T^{*} X\right.$ ) is a principal symbol of $A$ satisfying $a(t v)=a(v)$ for all $t>0$ and for all $v \in T^{*} X$ such that $|v|^{\prime} \geq 1$, then

$$
\inf \{\|A+K\| ; K \in \mathfrak{K}\}=\sup \left\{|a(v)|, v \in S^{*} X\right\},
$$

where $S^{*} X$ denotes the co-sphere bundle, $S^{*} X=\left\{v \in T^{*} X ;|v|^{\prime}=1\right\}$.

Corollary 2. The mapping $\left.[A] \mapsto a\right|_{S^{*} X}$, for $A \in \Psi_{c l}^{0}(X)$ and $a$ as in the statement of Theorem [2 extends to a $C^{*}$-algebra isomorphism $\hat{\sigma}: \mathfrak{A}_{c l} / \mathfrak{K} \rightarrow C\left(S^{*} X\right)$.

Proof: That this defines an isometric $\mathrm{C}^{*}$-algebra homomorphism follows exactly as in the proof of Theorem 2 To prove that it is surjective, it is enough to show that its image contains the dense subspace $C^{\infty}\left(S^{*} X\right)$. Given $b \in C^{\infty}\left(S^{*} X\right)$, let $a \in C^{\infty}\left(T^{*} X\right)$ be such that (3) holds for some $R_{0}<1$. The proof of the surjectivity of the principal symbol in [9, shortly before Definition 18.1.21, shows that there exists $A \in \Psi_{c l}^{0}(X)$ for which $a$ is a principal symbol.

Theorem 3. If $A \in \Psi^{0}(X) \cap \mathfrak{A}_{c l}$ then every principal symbol a of $A$ satisfies:

$$
\lim _{t \rightarrow+\infty} a(t v) \text { exists for all } v \in S^{*} X \text {. }
$$

Proof: Let us show that $\lim _{t \rightarrow+\infty} a(t v)=a_{0}(v)$, for $a_{0}=\hat{\sigma}([A])$ ( $\hat{\sigma}$ was defined in the statement of Corollary 21).

Let $t_{\alpha}>0$ be a net such that $\lim t_{\alpha}=+\infty$. Since $\mathbb{M}_{h}$ is compact, the net $t_{\alpha} v$ possesses a convergent subnet. Therefore, we may suppose that $t_{\alpha} v$ converges to $m_{0} \in \mathbb{M}_{s}$. We must have $m_{0} \notin T^{*} X$, because $\left|t_{\alpha} v\right| \rightarrow \infty$.

Let $A_{n}$ be a sequence in $\Psi_{c l}^{0}(X)$ converging to $A$, and let $a_{n}=\hat{\sigma}\left(\left[A_{n}\right]\right)$. By definition of $\hat{\sigma}, A_{n}$ has a principal symbol $\tilde{a}_{n}$ satisfying $\tilde{a}_{n}(t v)=a_{n}(v)$ for all $v \in S^{*} X$. By definition of $\sigma, \sigma\left(A_{n}\right)\left(m_{0}\right)=\lim _{\alpha} \tilde{a}_{n}\left(t_{\alpha} v\right)=a_{n}(v)$. Since $\sigma$ and $\hat{\sigma}$ are continuous, we get:

$$
\sigma(A)\left(m_{0}\right)=\lim _{n} \sigma\left(A_{n}\right)\left(m_{0}\right)=\lim _{n} a_{n}(v)=a_{0}(v),
$$

for $a_{0}=\hat{\sigma}([A])$. By definition of $\sigma$, and because $t_{\alpha} v \rightarrow m_{0}$,

$$
\sigma(A)\left(m_{0}\right)=\lim _{\alpha} a\left(t_{\alpha} v\right),
$$

and, hence,

$$
\lim _{t \rightarrow+\infty} a(t v)=a_{0}(v),
$$

if $a$ is a principal symbol for $A$.

To prove that $\mathfrak{A}_{c l}$ is strictly contained in $\mathfrak{A}$ (using Theorem 3 and the surjectivity of the principal symbol), it is therefore enough to give an example of a symbol in $S^{0}\left(T^{*} X\right)$ such that some of the radial limits in the statement of Theorem 3 fail to exist.

The author learned the following example from Jorge Hounie. 
Example Choose a non-negative $g \in C^{\infty}\left(\mathbb{R}^{n}\right)$, positive on $\{\xi ; 1<|\xi|<2\}$ and with support contained in $\{\xi ; 1 \leq|\xi| \leq 2\}$. It is not difficult to check that

$$
\psi(\xi)=\sum_{k=0}^{\infty} g\left(2^{-k} \xi\right)
$$

is a symbol in $S^{0}\left(\mathbb{R}^{n}\right)$ and that $\lim _{t \rightarrow \infty} \psi(t \xi)$ does not exist for any $\xi \neq 0$. If $\varphi$ is a smooth function with support contained in $\tilde{U}$, for some chart $\chi: U \rightarrow \tilde{U}$, then the pullback to $T^{*} X$ of $\varphi(x) \psi(\xi)$ by the bundle trivialization induced by $\chi$ gives an example of a symbol $a \in S^{0}\left(T^{*} X\right)$ for which $\lim _{t \rightarrow \infty} a(t v)$ does not exist for any $v \in T^{*} X$ such that $\varphi(\chi(\pi(v))) \neq 0$.

\section{4. $\mathfrak{A}_{c l}$ IS A COMPARISON ALGEBRA}

Let $\Delta$ denote the Laplace operator on a Riemannian manifold $\Omega$ equipped with the surface measure $d \mu$, and let $H$ denote the Friedrichs extension of $1-\Delta$ (this is a special self-adjoint realization on the Hilbert space $L^{2}(\Omega, d \mu)$ of the symmetric operator $1-\Delta$ with domain $C_{0}^{\infty}(\Omega)$ ). A comparison algebra for the triple $\{\Omega, d \mu, H\}$ was defined ${ }^{1}$ by Cordes (4, Chapter V) as the smallest norm-closed, adjointinvariant algebra of bounded operators on $L^{2}(\Omega, d \mu)$ containing:

(1) all operators of multiplication by elements of a class of smooth functions, and

(2) all operators of the form $L \Lambda$, where $\Lambda=H^{-1 / 2}$ and $L$ is an element of a class of first-order linear partial differential operators.

These classes of functions and of differential operators must then satisfy certain axioms.

In case $\Omega=X$ is compact and without boundary, those axioms imply that there is only one comparison algebra: it is the $\mathrm{C}^{*}$-algebra generated by all multiplications by smooth functions and by all operators of the form $L \Lambda$, where $L$ is any first-order linear differential operator with smooth coefficients. So, let $\mathfrak{C}$ denote the unique comparison algebra over $X$, defined by a chosen Riemannian metric. All generators of $\mathfrak{C}$ are classical pseudodifferential operators; hence $\mathfrak{C} \subseteq \mathfrak{A}_{c l}$.

Granted that $\mathfrak{C}$ contains the compact ideal $\mathfrak{K}$ (迆, Lemma V.1.1), the equality $\mathfrak{A}_{c l}=\mathfrak{C}$ is an easy consequence of following description of the Gelfand space and of the Gelfand mapping for the commutative $\mathrm{C}^{*}$-algebra $\mathfrak{C} / \mathfrak{K}$. This is a particular case of Theorem VI.2.2 in [4.

Theorem 4. The restriction of $\hat{\sigma}$ to $\mathfrak{C} / \mathfrak{K}$ is a $C^{*}$-algebra isomorphism.

Proof: In view of Corollary 2 all we have to show is that this restriction of $\hat{\sigma}$ is surjective. It is not difficult to show that the set of all functions

$$
a_{0}(v)=\lim _{t \rightarrow \infty} a(t v)
$$

where $a$ is a principal symbol of an $A=L \Lambda, L$ a smooth vector field, separates points on $S^{*} X$. The proof of Theorem 3 shows that $a_{0}=\hat{\sigma}([A])$. By the Stone-Weierstrass Theorem, the image of a subalgebra of $\mathfrak{C} / \mathfrak{K}$ is therefore dense in $C\left(S^{*} X\right)$.

\footnotetext{
${ }^{1}$ His definition is actually more general than this: the measure does not have to be the surface measure for some Riemannian metric, the elliptic operator does not have to be $1-\Delta$. He only requires that the operator and the measure be related by 4, (V.1.1).
} 
This shows that, for each $A \in \mathfrak{A}_{c l}$, there is $A^{\prime} \in \mathfrak{C}$ such that $A-A^{\prime}$ is compact. Since $\mathfrak{K} \subset \mathfrak{C}$, we then get $\mathfrak{A}_{c l}=\mathfrak{C}$.

\section{Acknowledgements}

The author was deeply influenced by Cordes' views about pseudodifferential operators, and about the $\mathrm{C}^{*}$-algebras generated by them, summarized in three books [3, 4, 5]. In particular, the main idea for this paper was borrowed from [5], Theorem V.10.3.

He also thanks Jorge Hounie and Elmar Schrohe for many conversations.

This work was partially supported by the Brazilian agency CNPq (Processo 300330/88-0).

\section{REFERENCES}

[1] M. F. АtiYah \& I. M. Singer, The index of elliptic operators I; Ann. of Math. (2) 87, 484-530, 1968.

[2] H. O. Cordes, On pseudodifferential operators and smoothness of special Lie-group representations; Manuscripta Math. 28, 51-69, 1979.

[3] H. O. Cordes, Elliptic Pseudodifferential Operators - An Abstract Theory; Lecture Notes in Mathematics 756, Springer, Berlin, 1979.

[4] H. O. Cordes, Spectral Theory of Linear Differential Operators and Comparison Algebras; London Mathematical Society Lecture Note Series 76, Cambridge University Press, Cambridge, 1987.

[5] H. O. Cordes, The technique of Pseudodifferential Operators; London Mathematical Society Lecture Note Series 202, Cambridge University Press, Cambridge, 1995.

[6] H. O. Cordes \& S. T. Melo, Smooth operators for the action of $S O(3)$ on $L^{2}\left(\mathbb{S}^{2}\right)$; Integr. Equat. Oper. Th. 28-3, 251-260 (1997).

[7] I. C. GohberG, On the theory of multidimensional singular integral operators; Dokl. Akad. Nauk SSR 133, 1279-1282, 1960.

[8] L. Hörmander, Pseudo-differential operators and hypoelliptic equations; Singular Integrals (Chicago, 1966), Proc. Symp. Pure Math. 10, 138-183, 1967.

[9] L. Hörmander, The Analysis of Linear Differential Operators III; Springer Verlag, Berlin, 1985.

[10] J. J. Kohn \& L. Nirenberg, An algebra of pseudo-differential operators; Comm. Pure Appl. Math. 18, 269-305, 1965.

[11] S. T. Melo, Smooth operators for the regular representation on homogeneous spaces; Studia Math. 142-2, 149-157, 2000.

[12] G. J. Murphy, C*-Algebras and Operator Theory; Academic Press, San Diego, 1990.

[13] K. R. Payne, Smooth Tame Fréchet Algebras and Lie Groups of Pseudodifferential Operators; Comm. Pure Appl. Math. 44, 309-337, 1991.

[14] M. Reed \& B. Simon, Methods of Modern Mathematical Physics I (Functional Analysis), Academic Press, New York, 1980.

[15] R. T. SeELey, Integro-differential operators on vector bundles; Trans. Amer. Math. Soc. 117, 167-204, 1965.

[16] M. E. TAYLOR, Beals-Cordes-type characterizations of pseudodifferential operators; Proc. Amer. Math. Soc. 125-6, 1711-1716, 1997.

[17] F. Treves, Introduction to Pseudodifferential and Fourier Integral Operators; Plenum Press, New York, 1980.

2000 Mathematics Subject Classification: 35S05 (58J40, 47G30).

Instituto de Matemática e Estatística, Universidade de São Paulo, Caixa Postal 66281, 05311-970 São Paulo, Brazil.

melo@ime.usp.br. 